\section{Description of polarisation dependence of two-photon absorption in silicon avalanche photodiodes}

\author{
J. O’Dowd, W.H. Guo, M. Lynch, E. Flood, A.L. Bradley \\ and J.F. Donegan
}

A simple formula is obtained that very accurately describes the level of two-photon absorption (TPA) generated by elliptically polarised light in silicon avalanche photodiodes (Si-APDs). The dichroism parameter necessary to describe the polarisation dependence of TPA in Si-APDs at room temperature is determined to be +0.372 in the region of $1550 \mathrm{~nm}$.

Introduction: Two-photon absorption (TPA) in semiconductors has recently generated strong interest as a means of performing a variety of optical pulse characterisation techniques. The autocorrelation of pulses as short as 6 fs has been reported using a TPA photodetector [1]. Optical sampling and channel identification in optical telecommunications networks have also been demonstrated using TPA detectors $[2,3]$. Previously, TPA based detection has been predominantly carried out using silicon avalanche photodiodes (Si-APDs). Owing to the fluctuation of signal polarisation in telecommunications networks it is essential to understand the polarisation dependence of TPA in SiAPDs very accurately so that these fluctuations can be properly accounted for in the pulse characterisation schemes. In a recent study, we have determined the polarisation dependence of TPA in a GaAs microcavity detector $[4,5]$.

In this Letter, we describe experiments to measure the polarisation dependence of TPA generated photocurrent $\left(I_{T P A}\right)$ in Si-APDs. We give a simple formula which very accurately describes this dependence at room temperature and show that it is not simply proportional to the optical field $(E)$ of the incident optical signal to the fourth power $I_{T P A} \propto\left\langle|E(t)|^{4}\right\rangle$, where $\langle$.$\rangle and |$.$| are the time average and magnitude$ of $E(t)$, respectively [6]. Also reported for the first time in Si-APDs at room temperature is the value of the dichroism parameter $(\delta)$ which is found to be +0.372 in the region of $1550 \mathrm{~nm}$.

Theory: The degenerate TPA generated photocurrent in Si can be described as $I_{T P A}=C \beta P(t)^{2}$, where $\beta$ is the polarisation-dependent TPA absorption coefficient in $\mathrm{Si}, C$ is a polarisation-independent constant, $P(t)$ is the power of incident optical signal which can be described as $\zeta \sqrt{P(t)}$ where $\zeta=\left[\begin{array}{c}\zeta_{a} \\ \zeta_{b}\end{array}\right]$ is the complex-valued Jones vector describing the signals polarisation. $\mathbf{a}, \mathbf{b}$ and $\mathbf{c}$ are unit vectors which define the local co-ordinate system. The signal polarisation and its propagation direction have been defined based on this local co-ordinate system. The polarisation dependence of degenerate TPA has previously been described in [7] as being dependent on three parameters. These are, $\chi_{x x x x}^{\prime \prime}, \sigma$ and $\delta$, where $\mathbf{x}, \mathbf{y}$ and $\mathbf{z}$ are the unit vectors along the [100], [010], and [001] crystallographic directions of $\mathrm{Si}$, respectively. $\chi$ is a fourth rank tensor that describes the third-order susceptibility of $\mathrm{Si}$, where $\chi_{x x x x}^{\prime \prime}$ is used as a shorthand representation of $\operatorname{Im}\left[\chi_{x x x x}^{\prime \prime}(\omega ;-\omega, \omega, \omega)\right]$, etc. $\sigma$ is the anisotropy parameter, where $\sigma=\left[\chi_{x x x x}^{\prime \prime}-\chi_{x x y y}^{\prime \prime}-2 \chi_{x y x y}^{\prime \prime}\right] / \chi_{x x x x}^{\prime \prime}$ and $\delta$ is the dichroism parameter, where $\delta=\left[\chi_{x x x x}^{\prime}+\chi_{x x y y}^{\prime}-2 \chi_{x y x y}^{\prime}\right] / \chi_{x x x x}^{\prime \prime}$. The value of $\sigma$ in Si-APDs has previously been reported at $1550 \mathrm{~nm}$ as 0 at room temperature $[6,8]$. The value for $\delta$ at a wavelength of $1550 \mathrm{~nm}$ at room temperature has not previously been reported in $\mathrm{Si}$.

The polarisation dependence of the TPA coefficient in $\mathrm{Si}$ has previously been described as $[5,7]$ :

$$
\begin{aligned}
\beta= & \frac{\omega}{2 n^{2} c^{2} \varepsilon_{0}} \chi_{x x x x}^{\prime \prime}\left(\left(1-\frac{\sigma}{2}-\delta\right)+\left(\delta-\frac{\sigma}{2}\right)\left|\zeta_{a}^{2}+\zeta_{b}^{2}\right|^{2}\right. \\
& \left.+\sigma\left(\left|\zeta_{a}\right|^{4}+\left|\zeta_{b}\right|^{4}\right)\right)
\end{aligned}
$$

where $\omega, n, c, \varepsilon_{o}$ are optical frequency, refractive index, speed of light and permittivity of free space, respectively. Based on (1) the TPA current generated in Si can be expressed in terms of $\sigma$ and $\delta$ :

$$
I_{T P A}=C^{\prime}\left(S_{0}(t)^{2}-\delta S_{3}(t)^{2}-\frac{\sigma}{2} S_{2}(t)^{2}\right)
$$

where $C^{\prime}=C \frac{\omega}{2 n^{2} c^{2} \varepsilon_{0}} \chi_{x x x x}^{\prime \prime}$ which is a polarisation-independent constant and $S_{0,2,3}$ are three Stokes parameters, where $S_{0}(t)=P(t)$,
$S_{2}(t)=\left(\zeta_{a} \zeta_{b}^{*}+\zeta_{a}^{*} \zeta_{b}\right) P(t)$, and $S_{3}(t)=-i\left(\zeta_{a} \zeta_{b}^{*}-\zeta_{a}^{*} \zeta_{b}\right) P(t)$. Using this definition, the TPA photocurrent generated by a polarised signal in Si-APDs can be easily calculated once $\sigma$ and $\delta$ are known.

Experiment: TPA measurements were carried out using a commercial (Perkin Elmer) Si-APD. A $1 \mathrm{~nm}$ band-limited ASE source (BL-ASE) was generated using an erbium-doped fibre amplifier (EDFA) with no injected optical signal. The ASE output passed through a $1 \mathrm{~nm}$ filter with its peak transmission centred on $1544 \mathrm{~nm}$. The BL-ASE passed through a polarisation controller which consists of an input polariser and quarter- and half-wave plates. Using the polarisation controller the polarisation of the input signal onto the detector can be set. The input signal was collimated and passed through a free space optical chopper before being focused onto the Si-APD using a 10x microscope objective (numerical aperture $=0.25$ ). It was ensured that the focused beam was normally incident on the detector. The Si-APD was biased at $180 \mathrm{~V}$, which generates sufficient current amplification so as to allow the TPA photocurrent signal to be monitored using a lock-in amplifier.

The TPA dependence on linear polarisation was investigated first. The incident power on the detector was kept constant at $1.94 \mathrm{~mW}$ for all polarisation measurements, which is high enough to ensure that TPA dominated the absorption over any single photon absorption process. The polarisation dependence of the TPA photocurrent was recorded as linear polarisation and was rotated around the equator of the Poincare sphere, the results are shown in Fig. 1. In this case, $S_{3}$ is zero, which as seen from (2), leads to the TPA dependence on linear polarisation orientation being independent of $\delta$. Fitting the linear polarisation dependence of the Si-APD allows $\sigma$ to be determined. The TPA photocurrent is found to be independent of linear polarisation orientation corresponding to $\sigma=0$, in line with previous reports $[6,8]$.

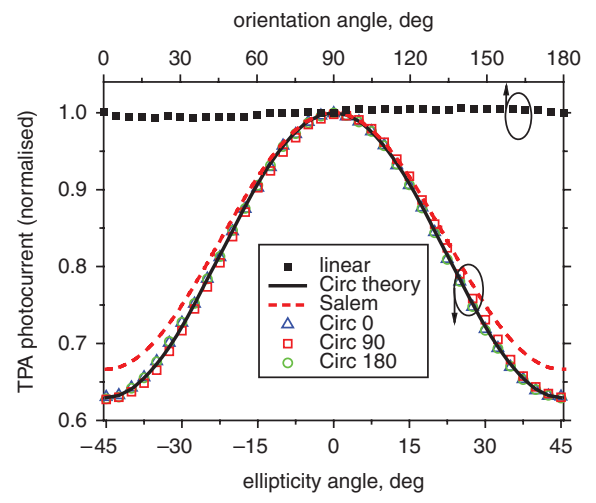

Fig. 1 Dependence of TPA photocurrent on state of polarisation Dependence recorded for linear case as polarisation is rotated around equator of Poincaré sphere. For elliptical polarisation case, polarisation is rotated from circular to linear to circular along three different longitudinal axes (Circ 0, 90, 180). TPA dependence on ellipticity fitted using equation given in this Letter (Circ theory) and approximation in [6] (Salem)

The polarisation dependence of the TPA photocurrent is then recorded as the input polarisation was rotated from circular to linear to circular along seven different longitudinal axes of the Poincaré sphere crossing the equator at orientation angles varying from $0^{\circ}$ to $180^{\circ}$ in steps of $30^{\circ}$, see Fig. 1 (where three representative curves are shown). From the curves it can be seen that the TPA photocurrent generated by a circularly-polarised optical signal is approximately 0.63 of that produced by a linearly polarised signal. The polarisation dependence is fitted using (2) with $\sigma=0$ and $\delta$ as the fitting parameter. The TPA data is normalised by the maximum TPA photocurrent value and then all TPA values are subtracted from 1 before being divided by $S_{3}^{2}$. TPA values associated with $S_{3}^{2}$ values less than 0.33 are excluded from the analysis owing to their proximity to the singularity associated with dividing by $S_{3}^{2}=0$ and the sensitivity of the fitting to small fluctuations associated with dividing by small $S_{3}^{2}$ values. The fitting results in a mean value for $\delta$ of +0.372 with a standard deviation of 0.001 associated with fitting the 168 remaining data points. This allows the polarisation dependence of TPA in Si-APDs around $1550 \mathrm{~nm}$ at room temperature to be expressed as:

$$
I_{T P A}=C^{\prime}\left(S_{0}(t)^{2}-0.372 S_{3}(t)^{2}\right)
$$


It can also be seen in Fig. 1 that the polarisation dependence of TPA calculated using the approximation in [6] does not result in as good an agreement with the ratio of TPA generated by linearly-compared with circularly-polarised signals

Conclusion: A simple equation is given to characterise the polarisation dependence of TPA in Si-APDs. This equation is shown to correctly take into account the influence of the dichroism parameter $(\delta)$. A value for $\delta$ of +0.372 around $1550 \mathrm{~nm}$ has been measured at room temperature and is shown to fit experimental data very well.

Acknowledgment: This work was supported by Science Foundation Ireland under its CSET, Centre for Telecommunications Value-chain Research and under its Research Frontiers Programme.

(C) The Institution of Engineering and Technology 2010

2 November 2009

doi: $10.1049 / \mathrm{el} .2010 .3090$

One or more of the Figures in this Letter are available in colour online.

J. O'Dowd, W.H. Guo, M. Lynch, E. Flood, A.L. Bradley and J.F. Donegan (CTVR, Semiconductor Photonics Group, School of Physics, Trinity College Dublin, Ireland)

E-mail: jodowd@tcd.ie

\section{References}

1 Ranka, J.K., Gaeta, A.L., Baltuska, A., Pshenichnikov, M.S., and Wiersma, D.A.: 'Autocorrelation measurement of 6-fs pulses based on the two-photon-induced photocurrent in a GaAsP photodiode', Opt. Lett., 1997, 22, (17), pp. 1344-1346
2 Kikuchi, K.: 'Optical sampling system at $1.5 \mu \mathrm{m}$ using two photon absorption in Si avalanche photodiode', Electron. Lett., 1998, 34, (13), pp. $1354-1355$

3 O’Dowd, J., Kilper, D.C., Guo, W.H., Donegan, J.F., and Chandrasekhar, S.: 'Optical channel monitoring using two photon absorption'. CLEOIQEC, Munich, Germany, 2007, pp. 1-1

4 Folliot, H., Lynch, M., Bradley, A.L., Dunbar, L.A., Hegarty, J., Donegan, J.F., Barry, L.P., Roberts, J.S., and Hill, G.: 'Two-photon absorption photocurrent enhancement in bulk AlGaAs semiconductor microcavities', Appl. Phys. Lett., 2002, 80, (8), pp. 1328-1330

5 O’Dowd, J., Guo, W.H., Flood, E., Lynch, M., Bradley, A.L., Barry, L.P., and Donegan, J.F.: 'Polarization dependence of a GaAs-based twophoton absorption microcavity photodetector', Opt. Express, 2008, 16, (22), pp. $17682-17688$

6 Salem, R., and Murphy, T.E.: 'Polarization-insensitive cross correlation using two-photon absorption in a silicon photodiode', Opt. Lett., 2004, 29, (13), pp. 1524-1526

7 Dvorak, M.D., Schroeder, W.A., Andersen, D.R., Smirl, A.L., and Wherrett, B.S.: 'Measurement of the anisotropy of two-photon absorption coefficients in Zincblende semiconductors', IEEE J. Quantum Electron., 1994, 30, (2), pp. 256-268

8 Kagawa, T., and Ooami, S.: 'Polarization dependence of two-photon absorption in Si avalanche photodiodes', Jpn. J. Appl. Phys., 2007, 46, (2), pp. 664-668 